\title{
Fluctuations in episodic and chronic migraine status over the course of 1 year: implications for diagnosis, treatment and clinical trial design
}

Daniel Serrano ${ }^{1 *}$, Richard B. Lipton ${ }^{2,3^{*}}$, Ann I. Scher ${ }^{4}$, Michael L. Reed ${ }^{5}$, Walter (Buzz) F. Stewart ${ }^{6}$, Aubrey Manack Adams ${ }^{7}$ and Dawn C. Buse ${ }^{8}$

\begin{abstract}
Background: Relatively little is known about the stability of a diagnosis of episodic migraine (EM) or chronic migraine (CM) over time. This study examines natural fluctuations in self-reported headache frequency as well as the stability and variation in migraine type among individuals meeting criteria for EM and CM at baseline.

Methods: The Chronic Migraine Epidemiology and Outcomes (CaMEO) Study was a longitudinal survey of US adults with EM and CM identified by a web-questionnaire. A validated questionnaire was used to classify respondents with EM ( $<15$ headache days/month) or CM ( $\geq 15$ headache days/month) every three months for a total of five assessments. We described longitudinal persistence of baseline EM and CM classifications. In addition, we modelled longitudinal variation in headache day frequency per month using negative binomial repeated measures regression models (NBRMR).

Results: Among the 5464 respondents with EM at baseline providing four or five waves of data, 5048 (92.4\%) had EM in all waves and 416 (7.6\%) had CM in at least one wave. Among 526 respondents with CM at baseline providing four or five waves of data, 140 (26.6\%) had CM in every wave and 386 (73.4\%) had EM for at least one wave. Individual plots revealed striking within-person variations in headache days per month. The NBRMR model revealed that the rate of headache days increased across waves of observation 19\% more per wave for CM compared to EM (rate ratio [RR], $1.19 ; 95 \% \mathrm{Cl}, 1.13-1.26)$. After adjustment for covariates, the relative difference changed to a $26 \%$ increase per wave (RR, 1.26; 95\% Cl, 1.2-1.33).

Conclusions: Follow-up at three-month intervals reveals a high level of short-term variability in headache days per month. As a consequence, many individuals cross the CM diagnostic boundary of $\geq 15$ headache days per month. Nearly three quarters of persons with CM at baseline drop below this diagnostic boundary at least once over the course of a year. These findings are of interest in the consideration of headache classification and diagnosis, the design and interpretation of epidemiologic and clinical studies, and clinical management.
\end{abstract}

Keywords: Migraine, Chronic migraine, Episodic migraine, Longitudinal, Remission

\footnotetext{
*Correspondence: daniel.serrano@endpointoutcomes.com;

Richard.Lipton@einstein.yu.edu

Daniel Serrano and Richard B. Lipton are co-first authors.

'Endpoint Outcomes, Boston, MA, USA

${ }^{2}$ The Saul R. Korey Department of Neurology, Albert Einstein College of

Medicine, Bronx, NY, USA

Full list of author information is available at the end of the article

The Enrico Greppi 2017 Selection Committee is:

Messoud Ashina, Anne Ducros, Antoinette Maassen van den Brink, Paolo

Martelletti, Francesco Pierelli, Massimiliano Valeriani
} 


\section{Background}

Episodic migraine (EM) and chronic migraine (CM) are primarily differentiated by monthly headache days, with EM having fewer than 15 headache days per month and $\mathrm{CM}$ having 15 or more headache days per month for at least three months [1]. The epidemiologic profiles of people with EM and CM are well characterized [2-5], as are annual rates of $\mathrm{CM}$ remission and $\mathrm{CM}$ onset from 1 year to the next [6]. However, relatively little is known about the longitudinal within-person variation in selfreported headache frequency when assessed repeatedly over the course of 1 year.

Migraine can be characterized as a chronic disorder with episodic attacks [7]. Within-person variation in headache days per month logically influences the epidemiologic estimates of EM and $\mathrm{CM}$ incidence and persistence reported in the literature [2-5], as well as the assessment of treatment response. However, epidemiology studies, for the most part, have evaluated incidence, persistence, remission, and disease progression by examining only two adjacent points in time. Furthermore, typically, only group mean data are reported. The natural within-person variation in headache day frequency, which is the fundamental driver of the instability in diagnostic classification for EM and CM reported in the literature, has been studied inadequately, if at all. Understanding of this natural history has been obscured in epidemiologic studies because of infrequent repeated measures and the emphasis on aggregated rather than within-person data.

To better characterize within-person change in headache days we analyzed longitudinal data from the Chronic Migraine Epidemiology and Outcomes (CaMEO) Study [8]. We examined transitions from EM to CM and from $\mathrm{CM}$ to EM. Of primary importance, we modelled natural history of self-reported headache day variation in quarterly assessments across the span of 15 months. Natural history of headache frequency was modeled accounting for both within-person and between-person changes in headache days per month. These findings have implications for classification and diagnosis, the design and conduct of observational studies, and for the design, management, and interpretation of randomized controlled trials (RCTs).

\section{Methods}

\section{CaMEO study design and baseline survey}

The CaMEO Study used a web-based panel (Research Now) constructed to be demographically representative of the US population, as previously detailed [8]. The baseline survey, e-mailed to participants, assessed headache symptoms and severity, headache frequency, headache-related disability, healthcare consulting and utilization, medication use for headache, comorbid medical and psychiatric conditions, and family-related burden associated with headache (Fig. 1).

\section{Ethics, consent and permissions}

Independent ethics committee approval was obtained before initiation of the CaMEO Study. Given that participation by individuals in the web-based survey was voluntary and that survey research does not expose respondents to known health risks, informed consent was not requested. No person-level identifiable data are included in this study report.

\section{Study classifications of EM and CM}

Respondents were identified as having migraine if they met modified International Classification of Headache Disorders Third edition (beta version) (ICHD-3 [beta]) criteria [1]. Among those with migraine, respondents were classified as having CM if they reported $\geq 15$ headache days per month averaged over the preceding 90 days (i.e., the Silberstein-Lipton approach [9]), otherwise they were classified as having EM. This definition of CM differs from standard ICHD-3 (beta) criteria by not requiring that headaches on eight of the 15 headache days per month be migraine (Criterion C).

\section{Questionnaire}

Of the 58,418 respondents to the baseline survey, 16,789 met criteria for EM or CM and received followup surveys at three-month intervals from September 2012 to November 2013. The baseline and four quarterly follow-up assessments constituted the five quarterly waves of data collection in the CaMEO Study, as previously described [8].

Headache frequency was assessed in all five waves using a question from the MIDAS questionnaire about the number of headache days over the last three months [10]. The three-month estimate was rescaled to a monthly estimate by dividing by three, paralleling the approach taken in the American Migraine Prevalence and Prevention (AMPP) study [3]. A diagnosis of EM or $\mathrm{CM}$ was assigned at each quarterly assessment. In addition, a continuous measure of headache days per month was retained to model variation in headache days within- and between-persons.

Sociodemographic covariates assessed at baseline included sex (female vs. male), employment status (full-time/part-time employment vs. other), education (college graduate or higher vs. less than college), race (Caucasian vs. non-Caucasian), Hispanic ethnicity (Hispanic vs. not), health insurance status (having health insurance vs. not), household income at or above the national median, and personal income at or above the national median. The two income variables were coded based on median income estimates obtained from the 


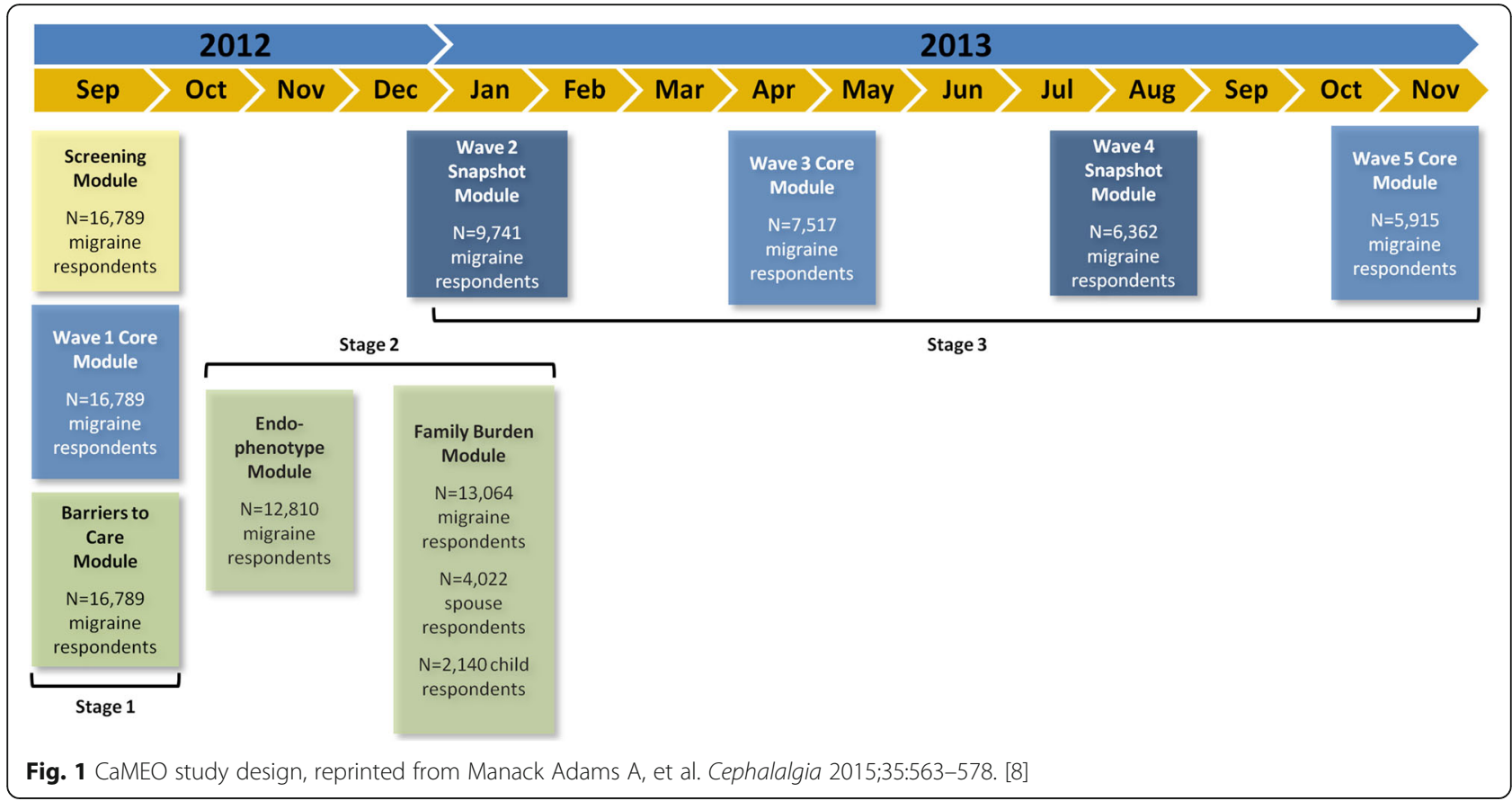

2012 American Community Survey conducted by the National Census Bureau [11].

In addition, the Migraine Disability Assessment questionnaire (MIDAS) categories were used as a disability covariate, with categories of zero to five indicating no disability (Grade I), six to 10 indicating mild disability (Grade II), 11-20 indicating moderate disability (Grade III), 21-40 indicating severe disability (Grade IV-A), and $\geq 41$ indicating very severe disability (Grade IV-B) [4]. A count of the self-report of physician diagnosis of comorbid conditions was used to generate a comorbidity index; included conditions were asthma, diabetes, high blood pressure, overactive bladder, shingles or herpes zoster, and post-herpetic neuropathy, with a count range of zero (no comorbidities) to six (all comorbidities). Lastly, age at migraine onset reported at baseline and migraine duration (age at baseline minus reported age at migraine onset) were included as disease duration covariates.

The longitudinal wave-specific participation rates for the total sample and stratified by baseline EM and CM status have been presented previously [8]; rates of participation were nearly identical between baseline EM and $\mathrm{CM}$ over time.

\section{Analyses}

Analysis first measured longitudinal persistence of baseline EM and CM classification, while accounting for attrition patterns in participation [8]. Analysis was also completed to model variation in average monthly headache days using the longitudinal negative binomial mixed effect models. Orthogonal polynomial terms were used to characterize the longitudinal variation in repeated measures trends and random effects were employed to account for subject-level variance in repeated measures. Technical details of the model building process are available in a separate document. Interested readers may request this document from the first author.

After determining the best-fitting mixed-effect polynomial parameterization, two primary models were fit. The first was an unadjusted model that included only the repeated measures trend terms and main effects and interactions between the repeated measures trend terms and $\mathrm{EM} / \mathrm{CM}$ status (with EM as reference). The second model adjusted the first with the incorporation of demographic characteristics as well as MIDAS disability, comorbidity count, and disease duration covariates. For the adjusted models, EM and CM status varied over time while all other covariates were entered as predictors based on their baseline status. The GLIMMIX procedure as implemented in SAS (Version 9.2) was used to estimate negative binomial models. All available data were used. Respondents with baseline data only contributed to the estimation of the intercept only. Respondents with more than a single wave of data contributed to estimates of longitudinal trends.

\section{Results}

Previously presented data [8] on the sampling design and participation rates will be reviewed to contextualize our findings. Sampling response, attrition, and cleaning rates have been published previously (see Table 2 in Manack et al. 2015) [8]. Of the 16,789 respondents with 
EM or CM at baseline, (wave one), 10,023 (59.7\%) responded and 9741 provided clean and useable returns (58.0\% of the baseline sample) at wave two. For subsequent waves, data are available for 7517 participants at wave three, 6363 participants for wave four and 5915 participants at wave five (Fig. 2). The attrition rate decreased with increasing follow-up.

The stability in headache diagnosis was examined descriptively by evaluating the proportion of individuals whose headache diagnosis was maintained across all waves (Table 1). The variation in headache diagnosis was examined by evaluating the proportion of individuals who had at least one wave with the alternative diagnosis. Among the 5464 respondents with EM at baseline providing four or five waves of data, 5048 (92.4\%) had EM at all waves and $416(7.6 \%)$ had CM in at least one wave. Among 526 respondents with $\mathrm{CM}$ at baseline providing four or five waves of data, 140 had CM at every wave (26.6\%) and 386 (73.4\%) had EM for at least one wave.

We modeled headache days per month at three-month intervals using linear, quadratic and cubic polynomial trends for time as well as measures of headache status. In these models, each individual subject is a random effect while the fixed effects represent average trends. As seen in Table 2, relatively large random-effect variances were observed for the polynomial trend terms, including the intercept (0.82), linear trend (0.17), and quadratic trend (0.09), as well as the negative binomial scaling variance parameter (0.09). These random effects reflect the substantial individual variation in monthly headache days. Plots of headache days at an individual-level revealed substantial subject-specific differences and striking curvatures (Additional file 1: Figure. S1). In addition, many fixed-effect estimates were statistically significant. The significant linear trend by headache status interaction indicated that headache days per wave changed differently for those with EM vs CM. Headache days per wave decreased slightly in the EM group and increased slightly in the CM group. This difference in change resulted in a rate of headache day increase of $19 \%$ more per wave for CM compared to EM (rate ratio [RR], 1.19; 95\% CI 1.13-1.26).

In Table 3, we augmented the unadjusted model with a set of covariates which diminished the random-effect variance estimates compared with the unadjusted model, particularly the baseline variance (intercept random effect). After adjustment, the random effect variance estimates for the intercept was 0.52 , with limited or no reductions in the linear trend variance (0.15), quadratic trend variance (0.09), and the negative binomial scaling variance (0.08) (Table 3 ). Nearly all fixed effects were statistically significant in the final model. After adjustment, the headache status by linear trend interactions revealed that headache days per month increased $26 \%$ more per wave for CM compared to EM (RR, 1.26; 95\% CI, 1.20-1.33). Figure 3 demonstrates how closely predicted values reproduced observed variation and oscillation for subjects. Although the predicted values

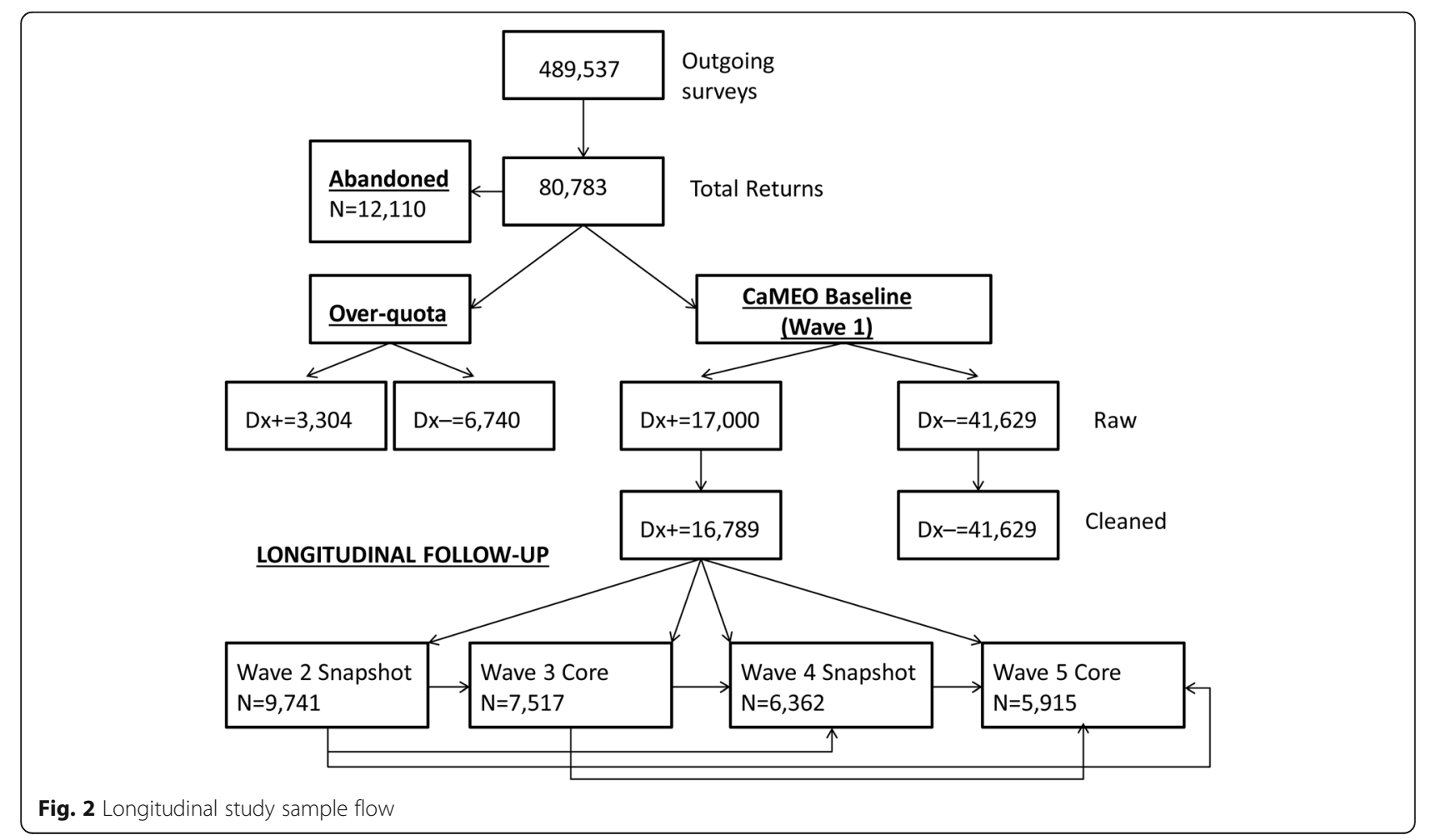


Table 1 Baseline headache status stability stratified on waves of study participation

\begin{tabular}{|c|c|c|c|}
\hline Waves participated & Number of waves baseline headache status maintained & $\begin{array}{l}\text { EM at Baseline, } \mathrm{n}(\%)^{\mathrm{a}} \text {; } \\
n=15,313\end{array}$ & $\begin{array}{l}\text { CM at Baseline, } \mathrm{n}(\%)^{\mathrm{a}} ; \\
n=1476\end{array}$ \\
\hline \multicolumn{4}{|c|}{ Respondents participating in 1 wave only (EM, $n=4801$; CM, $n=474)$} \\
\hline 1 & 1 & $4801(100)$ & $474(100)$ \\
\hline \multicolumn{4}{|c|}{ Respondents participating in any 2 waves (EM, $n=2839 ; C M, n=270$ ) } \\
\hline 2 & 1 & $99(3.5)$ & $159(58.9)$ \\
\hline 2 & 2 & $2740(96.5)$ & $111(41.1)$ \\
\hline \multicolumn{4}{|c|}{ Respondents participating in any 3 waves (EM, $n=2209 ; C M, n=206$ ) } \\
\hline 3 & 1 & $29(1.3)$ & $98(47.6)$ \\
\hline 3 & 2 & $98(4.4)$ & $53(25.7)$ \\
\hline 3 & 3 & $2082(94.3)$ & $55(26.7)$ \\
\hline \multicolumn{4}{|c|}{ Respondents participating in any 4 waves (EM, $n=2161 ; C M, n=206$ ) } \\
\hline 4 & 1 & $12(0.6)$ & $87(42.9)$ \\
\hline 4 & 2 & $31(1.4)$ & $34(16.7)$ \\
\hline 4 & 3 & $106(4.9)$ & $34(16.7)$ \\
\hline 4 & 4 & $2012(93.1)$ & $48(23.6)$ \\
\hline \multicolumn{4}{|c|}{ Respondents participating all 5 waves $(E M, n=3303 ; C M, n=323$ ) } \\
\hline 5 & 1 & $21(0.6)$ & $101(31.3)$ \\
\hline 5 & 2 & $35(1.1)$ & $43(13.3)$ \\
\hline 5 & 3 & $46(1.4)$ & $32(9.9)$ \\
\hline 5 & 4 & $165(5)$ & $55(17)$ \\
\hline 5 & 5 & $3036(91.9)$ & $92(28.5)$ \\
\hline
\end{tabular}

CM chronic migraine, EM episodic migraine

${ }^{\text {a }}$ ercentages are the percentages of the respondents participating in the identified number of waves

Table 2 Unadjusted model for headache days per month for persons with EM and CM

\section{Random-effect variance parameter estimates}

\section{Estimate}

Intercept

0.82

0.17

0.09

0.09

Scale

\section{Fixed Effects}

Label

Intercept

Linear trend

Quadratic trend

cubic trend

Headache status: CM vs. EM

Headache status by linear trend interaction

Headache status by quadratic trend interaction

Headache status by cubic trend interaction

\section{Standard Error}

0.01

0.01

0.01

0.005

$C M$ chronic migraine $D F$ degrees of freedom, $E M$ episodic migraine, $R R$ rate ratio, $t$ t-statistic

${ }^{\text {a }}$ Statistically significant RRs are bolded 
Table 3 Final adjusted and trimmed model for headache days per month ${ }^{\mathrm{a}}$

\begin{tabular}{|c|c|c|c|c|c|c|}
\hline \multicolumn{7}{|c|}{ Random-Effect Variance Parameter Estimates } \\
\hline & Estimate & \multicolumn{5}{|l|}{ Standard Error } \\
\hline Intercept & 0.52 & \multicolumn{5}{|l|}{0.01} \\
\hline Linear trend & 0.15 & \multicolumn{5}{|l|}{0.01} \\
\hline Quadratic trend & 0.09 & \multicolumn{5}{|l|}{0.01} \\
\hline Scale & 0.08 & \multicolumn{5}{|l|}{0.005} \\
\hline \multicolumn{7}{|l|}{ Fixed Effects ${ }^{b}$} \\
\hline \multicolumn{3}{|l|}{ Label } & $\operatorname{RR}(95 \% \mathrm{Cl})^{\mathrm{c}}$ & DF & t & $P$ value \\
\hline \multicolumn{3}{|l|}{ Intercept } & $1.16(1.02-1.32)$ & 16,650 & 2.28 & 0.0224 \\
\hline \multicolumn{3}{|c|}{ Sex: male vs. female } & $0.9(0.87-0.93)$ & 16,650 & -6.62 & $<0.0001$ \\
\hline \multicolumn{3}{|c|}{ Education: college graduate vs. non-college graduate } & $0.96(0.93-0.99)$ & 16,650 & -2.92 & 0.0035 \\
\hline \multicolumn{3}{|c|}{ Race: Caucasian vs. nonwhite } & $1.19(1.14-1.23)$ & 16,650 & 8.78 & $<0.0001$ \\
\hline \multicolumn{3}{|c|}{ Household income: at or above national median vs. those below } & $0.96(0.94-0.99)$ & 16,650 & -2.42 & 0.0155 \\
\hline \multicolumn{3}{|l|}{ Comorbid count } & $1.07(1.05-1.09)$ & 16,650 & 8.06 & $<0.0001$ \\
\hline \multicolumn{3}{|c|}{ Disability: MIDAS category TIC } & $1.47(1.46-1.49)$ & 16,650 & 73.56 & $<0.0001$ \\
\hline \multicolumn{3}{|l|}{ Age at onset, y } & $0.998(0.997-0.999)$ & 16,650 & -3.20 & 0.0014 \\
\hline \multicolumn{3}{|c|}{ Duration of illness } & $1.00(1.00-1.00)$ & 16,650 & 0.83 & 0.4090 \\
\hline \multicolumn{3}{|l|}{ Linear trend } & $0.74(0.73-0.76)$ & 29,304 & -28.63 & $<0.0001$ \\
\hline \multicolumn{3}{|l|}{ Quadratic trend } & $1.09(1.07-1.11)$ & 29,304 & 8.35 & $<0.0001$ \\
\hline \multicolumn{3}{|l|}{ Cubic trend } & $0.99(0.97-1.01)$ & 29,304 & -1.34 & 0.1797 \\
\hline \multicolumn{3}{|c|}{ Headache status: CM vs. EM } & $3.95(3.84-4.07)$ & 29,304 & 90.71 & $<0.0001$ \\
\hline \multicolumn{3}{|c|}{ Headache status by linear trend interaction } & $1.26(1.2-1.33)$ & 29,304 & 9.34 & $<0.0001$ \\
\hline \multicolumn{3}{|c|}{ Headache status by quadratic trend interaction } & $0.99(0.94-1.03)$ & 29,304 & -0.51 & 0.6434 \\
\hline \multicolumn{3}{|c|}{ Headache status by cubic trend interaction } & $1.00(0.96-1.04)$ & 29,304 & -0.17 & 0.8627 \\
\hline
\end{tabular}

$C M$ chronic migraine, DF degrees of freedom, EM episodic migraine, MIDAS Migraine Disability Assessment questionnaire, $R R$ rate ratio, $t \mathrm{t}$-statistic; TIC time invariant covariate, TVC time-varying covariate

an persons with EM and CM and adjusting for demographic variables, headache related disability and comorbidity

${ }^{b}$ All covariates were specified as TICs, though TVCs could have been specified for several including MIDAS and comorbidity count, which may not have been constant over time

'Statistically significant RR are bolded

are shrunken toward the mean relative to the observed values, they recover the observed value oscillations quite well. Figure 4 presents a plot of the EM- and CMspecific longitudinal trajectories characterizing this interaction. One can see that headache frequency for EM diminishes over time. In contrast, headache frequency oscillates for CM, but the overall trend is one of subtle increase. The reported rate ratios of 1.19 and 1.26 (adjusted) arise from this difference between headache frequency decreasing over time for EM while increasing over time for CM.

\section{Discussion}

This study demonstrates that in persons with migraine assessed at three-month intervals, there are frequent transitions between CM and EM with substantial withinperson variation in the number of headache days per month over the course of 15 months. Random-effect variance estimates demonstrated that there was significant temporal variability in headache days, both within and between the EM and CM populations. We observed statistically significant increases in headache frequency for $\mathrm{CM}$ relative to EM over time, both before and after adjustment for potential confounders. The high rates of transition between EM and CM have implications for headache classification and diagnosis, epidemiologic studies and clinical trial design.

\section{Implications for headache classification, diagnosis, and clinical practice}

We found that $73.4 \%$ of people with $\mathrm{CM}$ at baseline and four or five follow-up waves of data had at least 1 threemonth period when they did not meet the 15 or more headache day per month criteria for CM. Among persons with EM at baseline and four or five waves of follow-up, 7.6\% had at least one period when they met the headache frequency criteria for $\mathrm{CM}$. These findings suggest that over the course of 15 months, transitions 
OBSERVED VERSUS IBLUP (INVERSE LINK TRANSFORMED BEST LINEAR UNBIASED PREDICTOR) VALUES

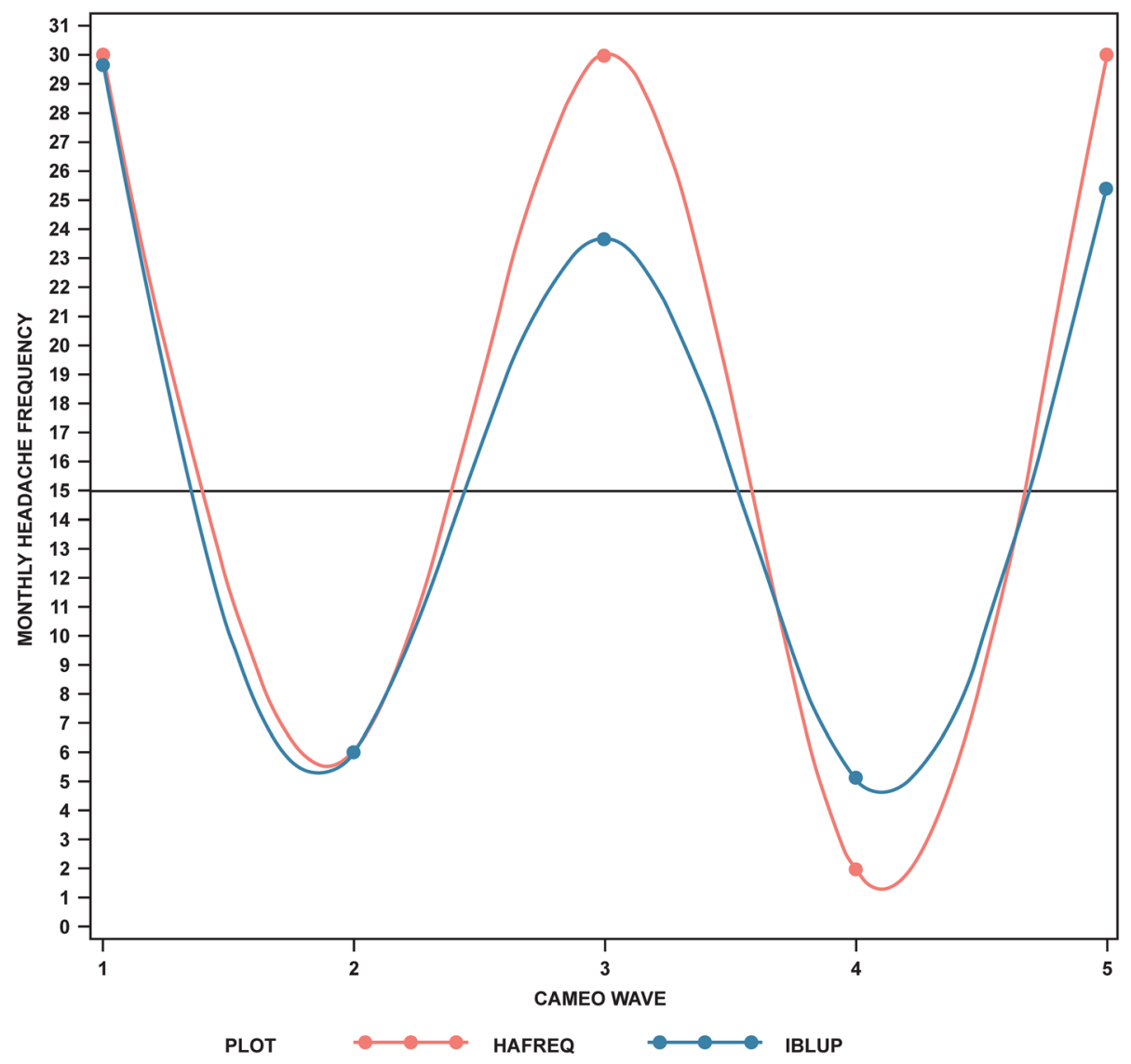

Fig. 3 Observed monthly headache frequency over 15 months compared with that predicted using IBLUP = approach HAFREQ = observed monthly headache frequency: IBLUP = inverse link function best linear unbiased predictor

between EM and CM are very common. In clinical practice, headache diagnosis is often assumed to be relatively stable for an individual, and yet we have found headache frequency changes substantially over time, potentially moving individuals between diagnostic categories (i.e., between EM and CM). When a person transitions from 16 headache days per month to 14 headache days per month, their ICHD-3 (beta) diagnostic label may change but the biology of their disorder most likely does not. The finding that headache day frequency within an individual changes substantially over time poses a considerable challenge to headache classification, diagnosis and subsequently clinical management. As diagnosis is intended to reflect a relatively stable set of clinical features and is used to predict treatment response and clinical course, this level of diagnostic instability is disconcerting. Perhaps headache days alone are not sufficiently stable to be the basis for distinguishing two related nosological entities such as EM and CM. We believe that there are more severe and less severe migraine biological subtypes of migraine, imperfectly differentiated by headache days criteria as EM and CM.
Future work could focus on identifying trait predictors of biotype, rather than time-varying features such as headache days per month.

When it comes to interpreting the effects of preventive treatment in practice, we often rely on a short-term reduction in headache days per month. The present findings suggest that at least part of the improvements we attribute to our treatments in practice may represent variation in headache days unrelated to treatment. This hypothesis requires further exploration by examining changes in headache days associated with stable treatment and with changes in treatment.

\section{Implications for epidemiologic studies}

Among people with EM at baseline, when assessed every three months, $7.6 \%$ of individuals met headache day criteria for $\mathrm{CM}$ at least once over a 15 -month period. This is much larger than the $2.5 \%$ rate of $\mathrm{CM}$ onset in persons with EM we reported after 1 year of follow-up in the AMPP study [12]. This apparent three-fold increased rate of $\mathrm{CM}$ onset in persons with $\mathrm{EM}$ with more frequent assessments, when coupled with the evidence of 


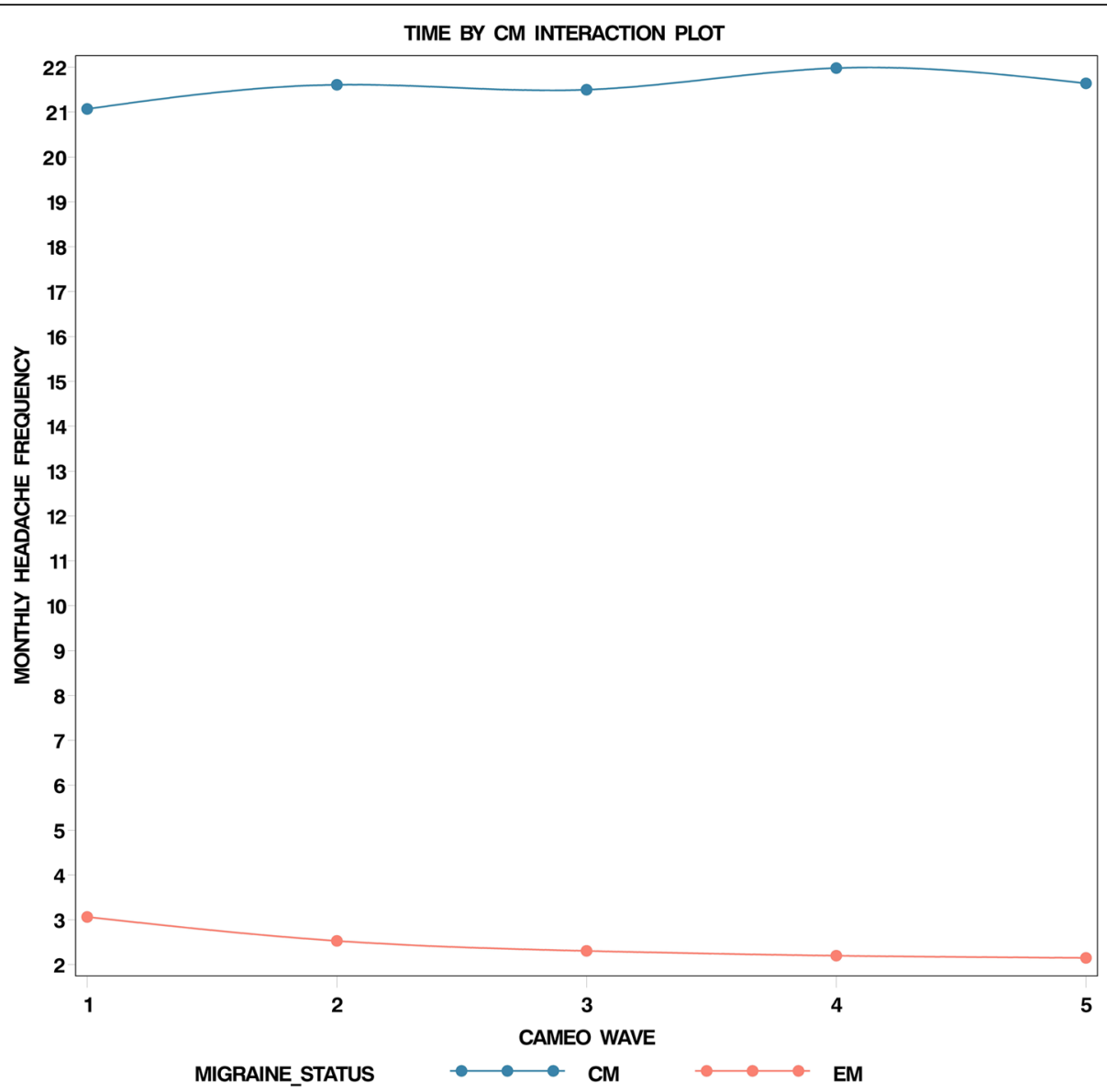

Fig. 4 Episodic- and chronic migraine-specific longitudinal trajectories. Time by chronic migraine interaction plot

transition from CM to EM, suggests that in studies using annual assessments, persons whose $\mathrm{CM}$ developed and remitted over the course of the year may be missed. Prior studies have also examined risk factors for $\mathrm{CM}$ $[3,13-16]$ and rates and risk factors for the remission of CM [12, 17, 18]. Previously reported factors associated with an increased risk of CM onset include headache features (such as cutaneous allodynia) [19], a broad array of comorbidities (including depression, allergic rhinitis, and asthma) [15] and poor acute treatment optimization among others [12]. Given the observed relative fluid and repeated movement across the "boundary" of 15 headache days per month in this systematically recruited sample of persons with migraine, the underlying framing of these studies may need to be reconsidered. The defining feature of $\mathrm{CM}$, the number of headache days per month over three months in a person with migraine, may reflect a process of continuous change within a single classification of migraine and not a change in the classification of disease as implied by the previous framing. It is likely that more frequent sampling will identify both higher rates of $\mathrm{CM}$ onset and stronger associations between risk factors and this outcome.

\section{Implications for clinical trial design}

Studies of CM use symptom profiles and headache day frequency to determine participation eligibility [20]. In preventive treatment trials of $\mathrm{CM}$, individuals judged by a clinician to have CM typically enter into a four-week diary study prior to study enrollment. If individuals have $<15$ headache days per month they are deemed ineligible for enrollment into the study. If they have $\geq 15$ headache days per month they are likely to be eligible and offered enrollment into the study. The primary endpoint in prevention trials is typically the change from baseline in the number of headache days per 28-day period on active drug vs. placebo or an active comparator [20]. Change in headache day frequency is typically assessed across two periods (from baseline to follow-up). The spontaneous and rarely measured variation in headache days provides a backdrop for interpreting treatment effects in individuals. There is a substantial probability that persons with $\mathrm{CM}$ at baseline will experience reductions unrelated to treatment in headache days as illustrated by the data reported here. Enrolling people with headache days above a threshold may lead to regression to the mean and high "placebo rates" [20]. This in turn can lead to modest 
therapeutic gains when active drug and placebo are compared [21]. We suggest that the high rate of spontaneous reduction in headache day frequency in RCTs may have contributed to what is widely regarded as an inescapably large placebo effect. This high presumed placebo effect observed in several studies may simply reflect the cyclic nature of CM [22-25].

When the results of our analysis (Fig. 3) are considered, one would expect that a majority of subjects selected at any high monthly headache-frequency value would, within three months, have a period of low headache-frequency suggesting that the high placebo rate may be, at least in part, an artifact of inclusion criteria interacting with the natural history of headache frequency. To empirically evaluate this point, we took the data from the CaMEO Study analyzed here, and looked at two waves of data. In the next three paragraphs we demonstrate that by selecting two waves of data and computing change in headache frequency between the two waves, headache frequency decreases for $\mathrm{CM}$ in a context where models fit to all waves demonstrated that frequency actually increased for CM. This calls into question the interpretability of two time-point change computations for headache frequency outcomes in both epidemiology studies and clinical trials.

Change in one-month recall of headache frequency was compared across two pairs of complete data from the CaMEO Study waves. The first pair was composed of waves one and two, which, with three months between them, directly mimics the assessment interval for the placebo arm in many prophylaxis trials. The second pair assessed longer-term change and was composed of waves one and five. Two variables were considered: (1) raw headache frequency and (2) change in headache frequency (headache frequency reported in the follow-up wave minus that reported in wave one). The effect of interest was whether change in headache frequency across the waves was different for EM and CM. Descriptive statistics, including wave EM- and CM-specific sample sizes and means for these three variables are given in Table 4. For the wave-specific headache frequency variables, a repeated measures negative binomial model was parameterized to calculate the difference between waves and estimate the difference in this difference between EM and CM through a time-by-headache status interaction term. The resulting time-by-headache status interaction RR emerging from this model along with the corresponding 95\% CI and $P$ values are reported in Table 4. This model was employed because it matches the modeling framework for the other models reported in this paper. For the variable in which change from baseline was calculated, a simple one-way analysis of variance (ANOVA) was conducted in which the only effect was baseline headache status. This model estimated the mean difference in change in headache frequency between baseline and follow-up between EM and CM, treating EM as the reference group. The resulting mean difference and corresponding 95\% CI and $P$ values are reported in Table 4.

In both cases (wave one and two pairing or wave one and five pairing), the results were identical. It appears that no matter what pairing of waves is used, headache frequency significantly decreases for $\mathrm{CM}$, while headache frequency for EM remains mostly constant. The change in headache frequency for $\mathrm{CM}$ was so great that at follow-up the average headache frequency would have resulted in the average population being classified as having achieved CM remission in both wave pairings through the natural history of the condition alone. The reported RRs for the time-by-baseline headache status interaction and the ANOVA mean difference support this conclusion and indicate that the $\mathrm{CM}$ reduction in headache frequency between wave pairings was statistically significantly greater than that of EM.

Results presented here suggest that when subject selection is made on the basis of high frequency of headache days at baseline, as a direct consequence of the cyclical nature of monthly headache day frequency, there is a nontrivial probability that within a short period of time, headache frequency will organically fall to lower levels. However, and most importantly, headache day frequency does not remain low, and for a sizeable number of people, headache frequency values return to even higher levels. This appears as a fundamental paradox: in the full data headache frequency for $\mathrm{CM}$ increases over time while comparisons between pairs of waves suggest that headache frequency decreases for CM. This paradox is related to a variant of the Yule-Simpson effect [26], where conclusions are distorted by specific binning or grouping of the data that is not representative of the true global trend in the phenomenon. Results presented in this manuscript would suggest that variation in headache frequency is substantial. Moreover, the practice in RCTs of restricting attention to a baseline period obtained through selection based on a high frequency of headache days in the baseline period coupled with examination of only a single subsequent follow-up period leads to the frequently observed placebo effect for $\mathrm{CM}$ prophylaxis trials. The use of more sensitive and sophisticated designs and analyses may enable more accurate characterization of longitudinal headache epidemiology and determine with greater certainty the efficacy of treatment.

\section{Strengths and limitations}

This study has a number of strengths and limitations. Strengths include the large sample size with inclusion of large numbers of persons with migraine, the three-month 


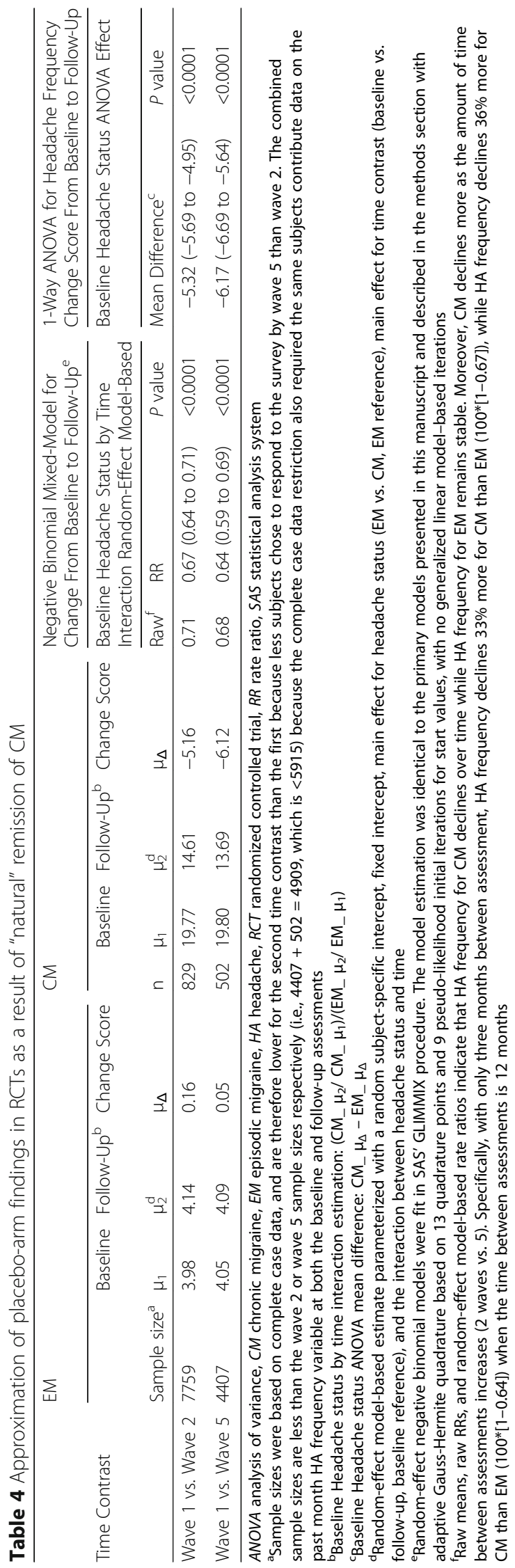


follow-up interval over a period of 15 months and our well-accepted modeling strategy. Limitations include the modest participation rate and the relatively high drop-out rate from wave to wave. The modest participation rate is offset in part by the evidence that results from the CaMEO Study are largely comparable to those from the larger AMPP study [6] and the non-responder study that did not provide any suggestion of participation bias [8]. As headache frequency varies over longer periods, modeling headache days using control theory-based models may provide better long-term characterization of the natural history of migraine across multiple intervals. Furthermore, the dynamic nature of migraine (transitioning between EM and CM) needs to be considered when a categorical description (i.e., EM or CM) is captured at baseline or individual time points. For example, a person categorized as having EM at baseline may have been classified with CM previously.

In addition, this study used modified ICHD-3 criteria which did not confirm $\geq$ five lifetime migraine events (criterion A) or duration of attack untreated from four to $72 \mathrm{~h}$ (criterion B). Likewise, the definition of CM did not include criterion $C$ which states that migraine occurred $\geq 8$ days per month. Confirmation of these parameters is difficult to evaluate via self-report retrospective surveys and requires data collection via diary cards and physician interview to verify.

\section{Conclusions}

Results confirm that there is substantial variation in headache day frequency in people with EM and CM followed at three-month intervals. Transitions from EM to $\mathrm{CM}$ are more common (7.6\%) than previously observed when sampled less frequently; in addition, nearly $75 \%$ of people with CM will remit to EM at some point during a 12-month period. More research is required to more fully understand the implications of these findings. Importantly, this natural variation should be considered when designing epidemiologic and clinical trials and when clinicians diagnose, treat and study CM.

\section{Additional file}

Additional file 1: Figure S1. Variability of Headache Day Frequency for Individuals with A) Episodic Migraine and B) Chronic Migraine at Wave One. (PDF $1392 \mathrm{~kb}$ )

\footnotetext{
Abbreviations

AMPP: American Migraine Prevalence and Prevention; ANOVA: Analysis of variance; CM: Chronic migraine; EM: Episodic migraine; IBLUP: Inverse link function best linear unbiased predictor; ICHD-3 (beta): International Classification of Headache Disorders Third edition (beta version); MIDAS: Migraine Disability Assessment questionnaire; RCT: Randomized controlled trial; RR: Rate ratio; SAS: Statistical analysis system
}

\section{Acknowledgments}

This study was sponsored by Allergan plc (Dublin, Ireland). Editorial assistance was provided to the authors by Lee B. Hohaia, Pharm D, and Gerard P. Johnson, PhD, of Complete Healthcare Communications, LLC (Chadds Ford, PA, USA), a CHC Group company, and funded by Allergan plc (Dublin, Ireland). All authors met the ICMJE authorship criteria. Neither honoraria nor payments were made for authorship.

\section{Funding}

Allergan ple (Dublin, Ireland) provided funding for this study. An individual employee from Allergan, who is acknowledged as an author, was involved in the conception and design, interpretation of data, and revising the manuscript critically for important intellectual input; giving final approval for publication of manuscript.

\section{Availability of data and materials}

The dataset supporting the conclusions of this article are available at www.ClinicalTrials.gov.

ClinicalTrials.gov identifier: NCT01648530;

https://clinicaltrials.gov/ct2/results?term=NCT01648530\&Search=Search Date of registration: July 20, 2012; Enrolment of first patient: September 2012.

\section{Authors' contributions}

DS made substantial contributions to conception and design, and analysis and interpretation of data, drafting of manuscript and revising critically for important intellectual input; giving final approval for publication of manuscript; and agrees to be accountable for all aspects of the work. RBL made substantial contributions to conception and design, acquisition of data, and interpretation of data, drafting of manuscript and revising critically for important intellectual input; giving final approval for publication of manuscript; and agrees to be accountable for all aspects of the work. AIS made substantial contributions to acquisition of data and interpretation of data, revising critically for important intellectual input; giving final approval for publication of manuscript; and agrees to be accountable for appropriate portions of the work. MLR made substantial contributions to conception and design, analysis and interpretation of data, revising critically for important intellectual input; giving final approval for publication of manuscript; and agrees to be accountable for appropriate portions of the work. WFS made substantial contributions to acquisition of data and interpretation of data, revising critically for important intellectual input; giving final approval for publication of manuscript; and agrees to be accountable for appropriate portions of the work. AMA made substantial contributions to conception and design, acquisition of data, and interpretation of data, revising critically for important intellectual input; giving final approval for publication of manuscript; and agrees to be accountable for appropriate portions of the work. DCB made substantial contributions to conception and design, acquisition of data, and interpretation of data, revising critically for important intellectual input; giving final approval for publication of manuscript; and agrees to be accountable for appropriate portions of the work.

\section{Competing interests}

Daniel Serrano is an employee of Endpoint Outcomes. This work was initiated while he was serving as a consultant for Vedanta Research and completed while Associate Director of Psychometrics at Endpoint Outcomes. He has received support funded by Allergan plc, CoLucid, Endo Pharmaceuticals, GlaxoSmithKline, MAP Pharmaceuticals, Merck \& Co., Inc., NuPathe, Novartis, and Ortho-McNeil, via grants to the National Headache Foundation. In addition, he has received funding from Allergan plc, Astellas, Genenteck, Lumena, Neurocrine, Shire and Supernus. Richard B. Lipton serves on the editorial board of Neurology and as senior advisor to Headache. He has received research support from the NIH. He also receives support from the Migraine Research Foundation and the National Headache Foundation. He has reviewed for the NIA and NINDS, serves as consultant, advisory board member, or has received honoraria from: Alder, Allergan, Amgen, Autonomic Technologies, Avanir, Boston Scientific, Colucid, Dr. Reddy's, Electrocore, Eli Lilly, eNeura Therapeutics, GlaxoSmithKline, Merck, Pfizer, Teva, and Vedanta. He receives royalties from Wolf's Headache, 8th Edition, Oxford University Press, 2009 and Informa. He holds stock options in eNeura Therapeutics and Biohaven. Ann I Scher, in the past 12 months has received honoraria from and is an advisory board member for Allergan, Inc., receives grant support from CDMRP and CNRM, and is on the editorial boards of Cephalalgia and Pain Medicine. The Views expressed are those of the author (s) and do not necessarily reflect the official views of the Uniformed Services University of the Health Sciences or the Department of 
Defense. Michael Reed is Managing Director of Vedanta Research, which has received research funding from Allergan, Amgen, CoLucid, Dr. Reddy's Laboratories, Endo Pharmaceuticals, GlaxoSmithKline, Merck \& Co., Inc., NuPathe, Novartis, and Ortho-McNeil, via grants to the National Headache Foundation. Vedanta Research has received funding directly from Allergan for work on the CaMEO Study. Walter (Buzz) F. Stewart consulted for Amgen and received support for research from Astra-Zeneca. Aubrey Manack Adams is a full-time employee of Allergan plc and owns stock in the company. Dawn C. Buse in the past 12 months, has received grant support and honoraria from Allergan, Avanir, Amgen, and Dr. Reddy's Laboratories. She has served as a consultant for Eli Lilly. She is an employee of Montefiore Medical Center, which in the past 12 months, has received research support funded by Allergan, Alder, Avanir, CoLucid, Dr. Reddy's Laboratories, and Labrys via grants to the National Headache Foundation and/or Montefiore Medical Center. She is on the editorial board of Current Pain and Headache Reports, the Journal of Headache and Pain, Pain Medicine News, and Pain Pathways magazine.

\section{Publisher's Note}

Springer Nature remains neutral with regard to jurisdictional claims in published maps and institutional affiliations.

\section{Author details}

${ }^{1}$ Endpoint Outcomes, Boston, MA, USA. ${ }^{2}$ The Saul R. Korey Department of Neurology, Albert Einstein College of Medicine, Bronx, NY, USA. ${ }^{3}$ Montefiore Headache Center; Department of Neurology and Department of Epidemiology and Population Health, Albert Einstein College of Medicine, Bronx, NY, USA. ${ }^{4}$ Department of Preventive Medicine and Biometrics, Uniformed Services University of the Health Sciences, Bethesda, MD, USA. ${ }^{5}$ Vedanta Research, Chapel Hill, NC, USA. ${ }^{6}$ Sutter Health, Walnut Creek, CA, USA. ${ }^{7}$ Allergan plc, Irvine, CA, USA. ${ }^{8}$ Montefiore Medical Center, Department of Neurology, Albert Einstein College of Medicine, Bronx, NY, USA.

Received: 1 June 2017 Accepted: 14 July 2017

Published online: 04 October 2017

\section{References}

1. Headache Classification Committee of the International Headache Society (2013) The International Classification of Headache Disorders, 3rd edition (beta version). Cephalalgia 33:629-808. doi:10.1177/0333102413485658

2. Lipton RB, Stewart WF, Diamond S, Diamond ML, Reed M (2001) Prevalence and burden of migraine in the United States: data from the American Migraine Study II. Headache 41:646-657

3. Buse DC, Manack AN, Fanning KM, Serrano D, Reed ML, Turkel CC, Lipton RB (2012) Chronic migraine prevalence, disability, and sociodemographic factors: results from the American Migraine Prevalence and Prevention Study. Headache 52:1456-1470. doi:10.1111/j.1526-4610.2012.02223.x

4. Blumenfeld AM, Varon SF, Wilcox TK, Buse DC, Kawata AK, Manack A, Goadsby PJ, Lipton RB (2011) Disability, HRQoL and resource use among chronic and episodic migraineurs: results from the International Burden of Migraine Study (IBMS). Cephalalgia 31:301-315. doi:10.1177/ 0333102410381145

5. Payne KA, Varon SF, Kawata AK, Yeomans K, Wilcox TK, Manack A, Buse DC, Lipton RB, Goadsby PJ, Blumenfeld AM (2011) The International Burden of Migraine Study (IBMS): study design, methodology, and baseline cohort characteristics. Cephalalgia 31:1116-1130. doi:10.1177/0333102411410610

6. Lipton RB, Manack Adams A, Buse DC, Fanning KM, Reed ML (2016) A comparison of the Chronic Migraine Epidemiology and Outcomes (CaMEO) Study and American Migraine Prevalence and Prevention (AMPP) Study: demographics and headache-related disability. Headache 56:1280-1289

7. Bigal ME, Lipton RB (2008) Concepts and mechanisms of migraine chronification. Headache 48:7-15

8. Manack Adams A, Serrano D, Buse DC, Reed ML, Marske V, Fanning KM, Lipton RB (2015) The impact of chronic migraine: the Chronic Migraine Epidemiology and Outcomes (CaMEO) Study methods and baseline results. Cephalalgia 35:563-578. doi:10.1177/0333102414552532

9. Silberstein SD, Lipton RB, Sliwinski M (1996) Classification of daily and neardaily headaches: field trial of revised IHS criteria. Neurology 47:871-875

10. Buse DC, Manack AN, Serrano D, Reed ML, Varon S, Turkel CC, Lipton RB (2012) Headache impact of chronic and episodic migraine: results from the American Migraine Prevalence and Prevention study. Headache 52:3-17. doi:10.1111/j.1526-4610.2011.02046.x
11. National Census Bureau (2012) 2012 American Community Survey. US Census Bureau. https://www.census.gov/programs-surveys/acs/data/pums. html. Accessed 9 Feb 2017

12. Bigal ME, Serrano D, Buse D, Scher A, Stewart WF, Lipton RB (2008) Acute migraine medications and evolution from episodic to chronic migraine: a longitudinal population-based study. Headache 48:1157-1168. doi:10.1111/j. 1526-4610.2008.01217.x

13. Aamodt AH, Stovner L, Langhammer A, Hagen K, Zwart JA (2007) Is headache related to asthma, hay fever, and chronic bronchitis? The HeadHUNT Study. Headache 47:204-212

14. Linde M, Stovner LJ, Zwart JA, Hagen K (2011) Time trends in the prevalence of headache disorders. The Nord-Trondelag Health Studies (HUNT 2 and HUNT 3). Cephalalgia. doi:10.1177/0333102410391488

15. Wang SJ, Fuh JL, Lu SR, Juang KD (2007) Outcomes and predictors of chronic daily headache in adolescents: a 2-year longitudinal study. Neurology 68:591-596. doi:10.1212/01.wnl.0000252800.82704.62

16. Scher Al, Stewart WF, Liberman J, Lipton RB (1998) Prevalence of frequent headache in a population sample. Headache 38:497-506. doi:10.1046/j.15264610.1998.3807497.x

17. Stewart WF, Wood GC, Bruce C, Buse DC, Runken MC, Lipton RB (2011) Longitudinal change in migraine headache-days and indirect cost consequences. J Occup Environ Med 53:478-487

18. Lipton RB (2009) Tracing transformation: chronic migraine classification, progression, and epidemiology. Neurology 72:S3-S7

19. Manack A, Buse DC, Serrano D, Turkel CC, Lipton RB (2011) Rates, predictors, and consequences of remission from chronic migraine to episodic migraine. Neurology 76:711-718. doi:10.1212/WNL.0b013e31820d8af2

20. Silberstein S, Tfelt-Hansen P, Dodick DW, Limmroth V, Lipton RB, Pascual J, Wang SJ (2008) Guidelines for controlled trials of prophylactic treatment of chronic migraine in adults. Cephalalgia 28:484-495. doi:10.1111/j.1468-2982. 2008.01555.x

21. Aurora SK, Dodick DW, Turkel CC, DeGryse RE, Silberstein SD, Lipton RB, Diener HC, Brin MF (2010) OnabotulinumtoxinA for treatment of chronic migraine: results from the double-blind, randomized, placebo-controlled phase of the PREEMPT 1 trial. Cephalalgia 30:793-803. doi:10.1177/ 0333102410364676

22. Bigal ME, Edvinsson L, Rapoport AM, Lipton RB, Spierings EL, Diener HC, Burstein R, Loupe PS, Ma Y, Yang R, Silberstein SD (2015) Safety, tolerability, and efficacy of TEV-48125 for preventive treatment of chronic migraine: a multicentre, randomised, double-blind, placebo-controlled, phase $2 \mathrm{~b}$ study. Lancet Neurol 14:1091-1100

23. Dodick DW, Turkel CC, DeGryse RE, Aurora SK, Silberstein SD, Lipton RB, Diener HC, Brin MF (2010) OnabotulinumtoxinA for treatment of chronic migraine: pooled results from the double-blind, randomized, placebocontrolled phases of the PREEMPT clinical program. Headache 50:921-936. doi:10.1111/j.1526-4610.2010.01678.x

24. Silberstein S, Lipton R, Dodick D, Freitag F, Mathew N, Brandes J, Bigal M, Ascher S, Morein J, Wright P, Greenberg S, Hulihan J (2009) Topiramate treatment of chronic migraine: a randomized, placebo-controlled trial of quality of life and other efficacy measures. Headache 49:1153-1162. doi:10.1111/j.1526-4610.2009.01508.x

25. Stovner LJ, Linde M, Gravdahl GB, Tronvik E, Aamodt AH, Sand T, Hagen K (2014) A comparative study of candesartan versus propranolol for migraine prophylaxis: a randomised, triple-blind, placebo-controlled, double crossover study. Cephalalgia 34:523-532

26. Blyth CR (1972) On Simpson's paradox and the sure-thing principle. J Am Stat Assoc 67:364-366. doi:10.2307/2284382 